MATHEMATICS OF COMPUTATION

Volume 75, Number 256, October 2006, Pages 1755-1778

S 0025-5718(06)01876-X

Article electronically published on July 6, 2006

\title{
RATE OF CONVERGENCE OF FINITE DIFFERENCE APPROXIMATIONS FOR DEGENERATE ORDINARY DIFFERENTIAL EQUATIONS
}

\author{
JIANFENG ZHANG
}

\begin{abstract}
In this paper we study finite difference approximations for the following linear stationary convection-diffusion equations:

$$
\frac{1}{2} \sigma^{2}(x) u^{\prime \prime}(x)+b(x) u^{\prime}(x)-u(x)=-f(x), \quad x \in \mathbb{R},
$$

where $\sigma$ is allowed to be degenerate. We first propose a new weighted finite difference scheme, motivated by approximating the diffusion process associated with the equation in the strong sense. We show that, under certain conditions, this scheme converges with the first order rate and that such a rate is sharp. To the best of our knowledge, this is the first sharp result in the literature. Moreover, by using the connection between our scheme and the standard upwind finite difference scheme, we get the rate of convergence of the latter, which is also new.
\end{abstract}

\section{INTRODUCTION}

Numerical methods for degenerate (elliptic or parabolic) PDEs, in particular degenerate HJB equations, have been studied by many authors. Notable works include: Markov chain approximation (e.g., Kushner-Dupuis 19]); viscosity solution method (e.g., Barles-Souganidis [3]); the works by Menaldi ([20]) and CamilliFalcone ([10]) on the so-called "control scheme"; Krylov's "shaking the coefficients" method ([16, [17]) and its extension by Barles-Jakobsen ([1]); the recent work by Bonnans-Zidani (4]); Karlsen's series of works, especially on the so-called entropy weak solutions to degenerate PDEs with discontinuous coefficients (e.g., 12, 9], [15]); as well as finite element methods such as the SPUG method (e.g., BrooksHughes [8]) and bubbles method (e.g., Brezzi et al. [5, 7], 6]), to mention a few. After this work was submitted for publication, we also learned that some new progresses were made on the subject (see, Jakobsen [13, Barles-Jakobsen [2, Krylov 18, and Dong-Krylov [11]).

Despite all these works, the rate of convergence of numerical approximations for degenerate PDEs is far from being fully understood. It is somewhat surprising that even for degenerate linear ODEs, there are no sharp results in the literature. To the best of our knowledge, the best rates are $h^{\frac{1}{3}}$ for finite difference schemes (see [16] or [1) and $h^{\frac{1}{2}}$ for control schemes (see [20]). The major difficulty lies in the

Received by the editor August 8, 2004 and, in revised form, June 27, 2005.

2000 Mathematics Subject Classification. Primary 65L70; Secondary 60H10, 65L12.

Key words and phrases. Degenerate convection-diffusion equations, finite difference approximations, probabilistic solutions, sharp rate of convergence.

The author was supported in part by NSF grant DMS-0403575.

(C)2006 American Mathematical Society 1755

Reverts to public domain 28 years from publication 
fact that, even if all the coefficients are infinitely smooth with compact support, the solutions to degenerate linear ODEs (not to mention fully nonlinear PDEs) are in general not sufficiently smooth.

We intend to understand how much one can expect to achieve on the rate of convergence of finite difference approximations for degenerate PDEs. Instead of being ambitious on generalization, in this paper we focus on the following 1-dimensional linear stationary convection-diffusion equations:

$$
\frac{1}{2} \sigma^{2}(x) u^{\prime \prime}(x)+b(x) u^{\prime}(x)-u(x)=-f(x), \quad x \in \mathbb{R},
$$

where, of course, $\sigma$ is allowed to be degenerate.

Our goal of the paper is twofold. We first propose a weighted finite difference scheme, motivated by some probabilistic approximation. Unlike classical Markov chain approximations which approximate the diffusion process associated with (1.1) in a weak or distributional sense (in other words, it matches the transition probabilities of the Markov chain with the coefficients of the finite difference equations, see, e.g., [19]), we try to approximate the diffusion process in a strong sense. Such an idea was also used by Menaldi [20. It turns out that these approximating processes can lead to probabilistic solutions of some finite difference equations, but with coefficients different from standard ones. We prove that, under certain conditions, our scheme converges with a rate of convergence $h$ and that such a rate is sharp.

We next study the standard upwind finite difference schemes. We find that the standard finite difference approximation to (1.1) is the same as our new weighted finite difference approximation to a new ODE with modified coefficients $\sigma_{h}, b_{h}$. We estimate the errors of $\sigma_{h}, b_{h}$ from $\sigma, b$, respectively, and then prove that this scheme converges with a rate of convergence $h^{\frac{1}{2}}$, and the rate becomes $h$ given some stronger conditions. The latter rate $h$ is also sharp, however, the sharpness of the general rate $h^{\frac{1}{2}}$ is still unknown.

At this point we should mention that our method takes advantage of some special properties of one dimension, and thus may be difficult to extend directly to highdimensional equations. However, besides the fact that this is the first sharp result in the literature, it provides a benchmark on what one can (or what one cannot) expect in high dimensions.

The rest of the paper is organized as follows. In $\S 2$ we introduce the two finite difference schemes and state the main results. In $\S \S 3$ and 4 we study the weighted and the standard finite difference schemes, respectively. In $\S 5$ we provide some counterexamples which show that some estimates in $\S 2$ are sharp. Finally some technical proofs are presented in the last section.

\section{MAin Results}

We first consider the following standard upwind finite difference approximation for (1.1):

$$
\begin{aligned}
& \frac{1}{2} \sigma^{2}(x) \frac{u_{h}^{s}(x+h)+u_{h}^{s}(x-h)-2 u_{h}^{s}(x)}{h^{2}}+(b(x) \vee 0) \frac{u_{h}^{s}(x+h)-u_{h}^{s}(x)}{h} \\
& \quad+(b(x) \wedge 0) \frac{u_{h}^{s}(x)-u_{h}^{s}(x-h)}{h}-u_{h}^{s}(x)=-f(x) .
\end{aligned}
$$

Here the superscript ${ }^{s}$ is the abbreviation for "standard". To simplify the presentation, throughout the paper we assume $b(x) \geq 0$. Then the above discrete equation 
can be rewritten as

$$
\begin{aligned}
& \frac{1}{2} \sigma^{2}(x) \frac{u_{h}^{s}(x+h)+u_{h}^{s}(x-h)-2 u_{h}^{s}(x)}{h^{2}}+b(x) \frac{u_{h}^{s}(x+h)-u_{h}^{s}(x)}{h}-u_{h}^{s}(x) \\
& \quad=-f(x) .
\end{aligned}
$$

Our first main result is the following theorem.

Theorem 2.1. Assume $\sigma, b$ are bounded and $\sigma, b, f \in C^{2}$ with bounded derivatives. Assume further that

$$
\left.L \triangleq \sup _{x}\left|2 b^{\prime}(x)+\right| \sigma^{\prime}(x)\right|^{2}\left|\vee \sup _{x}\right| b^{\prime}(x) \mid<1 .
$$

Then there exists a constant $C$, depending on $L$, the bounds of $\sigma, b$, and the bounds of the derivatives, such that

$$
\left|u_{h}^{s}(x)-u(x)\right| \leq C h .
$$

This rate is sharp.

We note that a condition similar to (2.2) was used by Menaldi 20. We also note that when the solution $u$ (not the coefficients!) is smooth enough, it is possible to obtain a much better rate.

In order to study a more general case where the coefficients are not in $C^{2}$, we adopt the following assumption:

(A) $\sigma, b$ are Lipschitz continuous and $f$ is bounded and Hölder- $\alpha$ continuous for some $\alpha \in(0,1]$.

Let $L_{b}$ denote the Lipschitz constant of $b$, and let $\beta>0$ be some arbitrary constant such that $\beta L_{b}<1$. Throughout the paper, we use a generic constant $C$, which may vary from line to line, to denote upper bounds of estimates which may depend on $\alpha, \beta$, the Lipschitz constants of $\sigma, b$, the Hölder constant of $f$, as well as some others specified in the context. We then have the following results.

Theorem 2.2. Assume (A).

(i) If $b$ is bounded, then

$$
\left|u_{h}^{s}(x)-u(x)\right| \leq C h^{\frac{1}{2}(\beta \wedge \alpha)} .
$$

(ii) If $\sigma=0$ or $\frac{b}{\sigma}$ is bounded (e.g., $b=0$ or $\sigma$ is uniformly nondegenerate), then we obtain the sharp rate

$$
\left|u_{h}^{s}(x)-u(x)\right| \leq C h^{\beta \wedge \alpha} .
$$

Remark 2.3. The sharpness of (2.4) is still unknown.

In order to prove Theorems 2.1 and 2.2, we introduce another weighted finite difference approximation for (1.1). This scheme is interesting in its own right, and is new to the best of our knowledge. To this end, we note that (2.1) is equivalent to

$$
u_{h}^{s}(x)=p_{h}^{s}(x) u_{h}^{s}(x+h)+q_{h}^{s}(x) u_{h}^{s}(x-h)+f(x)\left[1-p_{h}^{s}(x)-q_{h}^{s}(x)\right],
$$

where

$$
p_{h}^{s} \triangleq \frac{\sigma^{2}+2 b h}{2\left[\sigma^{2}+b h+h^{2}\right]} ; \quad q_{h}^{s} \triangleq \frac{\sigma^{2}}{2\left[\sigma^{2}+b h+h^{2}\right]} .
$$


By using the superscript ${ }^{w}$ to denote "weighted", we define our new scheme as follows:

$$
u_{h}^{w}(x)=p_{h}^{w}(x) u_{h}^{w}(x+h)+q_{h}^{w}(x) u_{h}^{w}(x-h)+f(x)\left[1-p_{h}^{w}(x)-q_{h}^{w}(x)\right],
$$

where

$$
\begin{aligned}
p_{h}^{w} & \triangleq \frac{1}{2} \exp \left(\frac{b h}{\sigma^{2}}\right)\left[\cosh \left(\frac{\sqrt{b^{2}+2 \sigma^{2}}}{\sigma^{2}} h\right)\right]^{-1}, \\
q_{h}^{w} & \triangleq \frac{1}{2} \exp \left(-\frac{b h}{\sigma^{2}}\right)\left[\cosh \left(\frac{\sqrt{b^{2}+2 \sigma^{2}}}{\sigma^{2}} h\right)\right]^{-1} .
\end{aligned}
$$

When $\sigma=0$, we take the limits of the above expressions as the values of $p_{h}^{w}$ and $q_{h}^{w}$. To be specific, we define that

$$
\left\{\begin{array}{lll}
p_{h}^{w} \triangleq \exp \left(-\frac{h}{b}\right), & q_{h}^{w} \triangleq 0, & \text { if } \quad b>0, \\
p_{h}^{w} \triangleq 0, & q_{h}^{w} \triangleq \exp \left(-\frac{h}{b}\right), & \text { if } \quad b<0, \\
p_{h}^{w} \triangleq 0, & q_{h}^{w} \triangleq 0, & \text { if } \quad b=0 .
\end{array}\right.
$$

We note that when $\sigma>0$, it holds that $\lim _{h \rightarrow 0} \frac{p_{h}^{w}}{p_{h}^{s}}=\lim _{h \rightarrow 0} \frac{q_{h}^{w}}{q_{h}^{s}}=1$. In this sense (2.8) and (2.6) are asymptotically equivalent, and thus one may expect that $u_{h}^{w}$ is also an approximation of $u$. This is indeed true.

Theorem 2.4. Assume (A). Then we have the following sharp estimate:

$$
\left|u_{h}^{w}(x)-u(x)\right| \leq C h^{\beta \wedge \alpha} .
$$

\section{The Weighted Finite DifFerenCE SCHEME}

In this section we shall prove (2.10) and leave the sharpness to $\S 5$. First, it is well known that the solution to (1.1) can be written as

$$
u(x)=E_{x}\left\{\int_{0}^{\infty} e^{-t} f\left(X_{t}\right) d t\right\},
$$

where $X$ is the solution to the following SDE:

$$
X_{t}=x+\int_{0}^{t} \sigma\left(X_{r}\right) d W_{r}+\int_{0}^{t} b\left(X_{r}\right) d r
$$

Here the subscript $x$ in $E_{x}$ indicates the fact that $X_{0}=x$, but for simplicity we shall omit it in the sequel when there is no confusion. The solution is unique in the sense that $u$ is bounded whenever $f$ is bounded. We refer the readers to [14] or [21] for basic theories of stochastic calculus.

The main idea for proving (2.10) is to find a similar probabilistic expression for $u_{h}^{w}$. We proceed by "freezing" the coefficients in (3.2). To this end, we fix $h$ and $x$, and construct a sequence of increasing stopping times $\tau_{n} \triangleq \tau_{n}^{h, x}$ and a process $X^{h} \triangleq X^{h, x}$ as follows. First we define $\tau_{0} \triangleq 0, X_{\tau_{0}}^{h} \triangleq x$. Then for $n=1,2, \cdots$, define

$$
\tau_{n} \triangleq \inf \left\{t>\tau_{n-1}:\left|\sigma\left(X_{\tau_{n-1}}^{h}\right)\left[W_{t}-W_{\tau_{n-1}}\right]+b\left(X_{\tau_{n-1}}^{h}\right)\left[t-\tau_{n-1}\right]\right|=h\right\},
$$


and for $t \in\left(\tau_{n-1}, \tau_{n}\right]$,

$$
X_{t}^{h} \triangleq X_{\tau_{n-1}}^{h}+\sigma\left(X_{\tau_{n-1}}^{h}\right)\left[W_{t}-W_{\tau_{n-1}}\right]+b\left(X_{\tau_{n-1}}^{h}\right)\left[t-\tau_{n-1}\right] .
$$

We note that these processes were also used by Menaldi [20. Since $\sigma$ and $b$ are Lipschitz continuous, hence grow at most linearly, one can easily show that $\tau_{n} \uparrow \infty$, almost surely. Denote $\tau(t) \triangleq \tau_{n-1}$ for $t \in\left[\tau_{n-1}, \tau_{n}\right)$. Then obviously it holds that

$$
\left|X_{\tau(t)}^{h}-X_{t}^{h}\right| \leq h, \quad \text { a.s. }
$$

and that

$$
X_{t}^{h}=x+\int_{0}^{t} \sigma\left(X_{\tau(s)}^{h}\right) d W_{s}+\int_{0}^{t} b\left(X_{\tau(s)}^{h}\right) d s .
$$

The following lemma gives the probabilistic solution to (2.8).

Lemma 3.1. Assume all the conditions in Theorem 2.4 hold true. Then

$$
u_{h}^{w}(x)=E_{x}\left\{\int_{0}^{\infty} e^{-t} f\left(X_{\tau(t)}^{h}\right) d t\right\} .
$$

Proof. Let $\bar{u}_{h}$ denote the right side of (3.7). Then

$$
\begin{aligned}
& \bar{u}_{h}(x)=E_{x}\left\{\left(\int_{0}^{\tau_{1}}+\int_{\tau_{1}}^{\infty}\right) e^{-t} f\left(X_{\tau(t)}^{h}\right) d t\right\} \\
& =E_{x}\left\{\int_{0}^{\tau_{1}} e^{-t} f\left(X_{\tau_{0}}^{h}\right) d t+\int_{0}^{\infty} e^{-\tau_{1}-s} f\left(X_{\tau^{h, X_{\tau_{1}}}(s)}^{h, X_{1}^{h}}\right) d s\right\},
\end{aligned}
$$

where $\tau^{h, X_{\tau_{1}}^{h}}$ and $X^{h, X_{\tau_{1}}^{h}}$ are defined in the same way as $\tau^{h}\left(=\tau^{h, x}\right)$ and $X^{h}\left(=X^{h, x}\right)$, with the initial value $x$ replaced by $X_{\tau_{1}}^{h, x}$. Note that $X_{\tau_{0}}^{h, x}=x$ and $X_{\tau_{1}}^{h, x}=x \pm h$. Then one can check directly that

$$
\bar{u}_{h}(x)=\bar{p}_{h}(x) \bar{u}_{h}(x+h)+\bar{q}_{h}(x) \bar{u}_{h}(x-h)+f(x)\left[1-\bar{p}_{h}(x)-\bar{q}_{h}(x)\right],
$$

where

$$
\bar{p}_{h}(x) \triangleq E_{x}\left\{e^{-\tau_{1}} 1_{\left\{X_{\tau_{1}}^{h}=x+h\right\}}\right\}, \quad \bar{q}_{h}(x) \triangleq E_{x}\left\{e^{-\tau_{1}} 1_{\left\{X_{\tau_{1}}^{h}=x-h\right\}}\right\} .
$$

So it suffices to show that

$$
\bar{p}_{h}(x)=p_{h}^{w}(x), \quad \bar{q}_{h}(x)=q_{h}^{w}(x) .
$$

Without loss of generality, we shall prove (3.10) only for $x=0$.

We note that by (3.3) and (3.4) one can calculate $\bar{p}_{h}$ and $\bar{q}_{h}$ straightforwardly (see, e.g., [14) and thus prove (3.10). But here we would like to provide another argument which avoids the probabilistic calculation. To this end, we consider the following ODE with constant coefficients:

$$
\frac{1}{2} \sigma^{2}(0) \bar{u}^{\prime \prime}(x)+b(0) \bar{u}^{\prime}(x)-\bar{u}(x)=-f(x) .
$$

By (3.1) we have

$$
\bar{u}(x)=E_{x}\left\{\int_{0}^{\infty} e^{-t} f\left(\bar{X}_{t}\right) d t\right\}
$$


where $\bar{X}$ satisfies

$$
\bar{X}_{t}=x+\int_{0}^{t} \sigma(0) d W_{r}+\int_{0}^{t} b(0) d r=x+\sigma(0) W_{t}+b(0) t .
$$

Note that $\bar{p}_{h}(0)$ and $\bar{q}_{h}(0)$ involve only $\sigma(0)$ and $b(0)$. Following the same arguments as for (3.8) we get, for the same $\bar{p}_{h}(0)$ and $\bar{q}_{h}(0)$ as in (3.9),

$$
\bar{u}(0)=\bar{p}_{h}(0) \bar{u}(h)+\bar{q}_{h}(0) \bar{u}(-h)+f(0)\left[1-\bar{p}_{h}(0)-\bar{q}_{h}(0)\right] .
$$

We note that (3.12) holds true for all $f$ and the corresponding solution $\bar{u}$. Choose $f$ such that $f(x)=0$ for $|x|<h$ and $f^{\prime}(h) \neq 0, f^{\prime}(-h) \neq 0$. Then in $(-h, h), \bar{u}$ satisfies the homogeneous ODE:

$$
\frac{1}{2} \sigma^{2}(0) \bar{u}^{\prime \prime}(x)+b(0) \bar{u}^{\prime}(x)-\bar{u}(x)=0 .
$$

Thus, for $x \in(-h, h)$,

$$
\bar{u}(x)=\alpha e^{\lambda_{1} x}+\beta e^{\lambda_{2} x},
$$

where $\alpha, \beta$ are two constants, and

$$
\lambda_{1} \triangleq \frac{-b(0)+\sqrt{b^{2}(0)+2 \sigma^{2}(0)}}{\sigma^{2}(0)}, \quad \lambda_{2} \triangleq \frac{-b(0)-\sqrt{b^{2}(0)+2 \sigma^{2}(0)}}{\sigma^{2}(0)} .
$$

Now by the continuity of $\bar{u}$ we have

$$
\bar{u}(h)=\alpha e^{\lambda_{1} h}+\beta e^{\lambda_{2} h}, \quad \bar{u}(-h)=\alpha e^{-\lambda_{1} h}+\beta e^{-\lambda_{2} h} .
$$

By straightforward calculation we get the values of $\alpha, \beta$ and check that

$$
\bar{u}(0)=\alpha+\beta=p_{h}^{w}(0) \bar{u}(h)+q_{h}^{w}(0) \bar{u}(-h) .
$$

Since $f(0)=0,(3.12)$ becomes

$$
\bar{u}(0)=\bar{p}_{h}(0) \bar{u}(h)+\bar{q}_{h}(0) \bar{u}(-h) .
$$

Compare (3.13) and (3.14), and note that both of them hold true for arbitrary values of $\bar{u}(h)$ and $\bar{u}(-h)$; we prove (3.10) at $x=0$, and hence the lemma.

To prove the theorem, we shall need another technical lemma. The arguments of the proof are mainly due to N. Krylov. We refer the readers to 14 or 21 again for preliminary materials.

Lemma 3.2. Let $X$ be the solution to the following linear SDE:

$$
X_{t}=x+\int_{0}^{t}\left(\alpha_{s} X_{s}+\beta_{s}\right) d W_{s}+\int_{0}^{t}\left(\gamma_{s} X_{s}+\lambda_{s}\right) d s
$$

where $\left|\alpha_{t}\right| \leq K_{1},\left|\gamma_{t}\right| \leq K_{2}$ for some $K_{1}, K_{2}>0$. Then for any $\varepsilon>0$, there exist constants $C$ and $q>2$, depending only on $K_{1}, K_{2}$ and $\varepsilon$, such that

$$
E\left\{\left|X_{t}\right|\right\} \leq C e^{\left(K_{2}+\varepsilon\right) t}\left[|x|+E\left\{\int_{0}^{t}\left[\left|\beta_{s}\right|^{q}+\left|\lambda_{s}\right|^{q}\right] d s\right\}^{\frac{1}{q}}\right], \quad \forall t \geq 0 .
$$

Moreover, if $\left|\beta_{t}\right|,\left|\lambda_{t}\right| \leq K_{3}$, then there exists $C$ which may depend on $K_{3}$ as well, such that

$$
E\left\{\left|X_{t}\right|\right\} \leq C e^{\left(K_{2}+\varepsilon\right) t}\left[|x|+K_{3}\right], \quad \forall t \geq 0 .
$$


Proof. Apply (3.15) with $\frac{\varepsilon}{2}$ and note that $t^{\frac{1}{q}} \leq C e^{\frac{\varepsilon}{2} t}$; one proves (3.16).

So it remains to prove (3.15). To this end, we denote

$d \tilde{W}_{t} \triangleq d W_{t}-\alpha_{t} d t, \quad M_{t} \triangleq \exp \left(-\int_{0}^{t} \alpha_{s} d W_{s}+\frac{1}{2} \int_{0}^{t} \alpha_{s}^{2} d s\right), \quad \Lambda_{t} \triangleq \exp \left\{-\int_{0}^{t} \gamma_{s} d s\right\}$.

By the Girsanov Theorem, $\tilde{W}$ is a Brownian motion under a new probability measure $\tilde{P}$ such that $\tilde{E}\left\{\xi_{t}\right\}=E\left\{M_{t}^{-1} \xi_{t}\right\}$ for any $\xi_{t} \in \mathcal{F}_{t}$. Applying Itô's formula one gets

$$
d\left(X_{t} M_{t} \Lambda_{t}\right)=M_{t} \Lambda_{t}\left[\beta_{t} d \tilde{W}_{t}+\lambda_{t} d t\right]
$$

Denote

$$
N_{t} \triangleq X_{t} M_{t} \Lambda_{t}=x+\int_{0}^{t} M_{s} \Lambda_{s} \beta_{s} d \tilde{W}_{s}+\int_{0}^{t} M_{s} \Lambda_{s} \lambda_{s} d s
$$

Then

$$
E\left\{\left|X_{t}\right|\right\}=\tilde{E}\left\{M_{t}\left|X_{t}\right|\right\}=\tilde{E}\left\{N_{t} \Lambda_{t}^{-1}\right\} .
$$

By the Ito formula again we have

$$
d\left(N_{t} \Lambda_{t}^{-1}\right)=\gamma_{t} N_{t} \Lambda_{t}^{-1} d t+M_{t} \beta_{t} d \tilde{W}_{t}+M_{t} \lambda_{t} d t .
$$

Thus, by noting that $\left|\gamma_{t}\right| \leq K_{2}$,

$$
\tilde{E}\left\{\mid N_{t} \Lambda_{t}^{-1}\right\} \leq|x|+K_{2} \int_{0}^{t} \tilde{E}\left\{\left|N_{s} \Lambda_{s}^{-1}\right|\right\} d s+\tilde{E}\left\{\left|\int_{0}^{t} M_{s} \beta_{s} d \tilde{W}_{s}+\int_{0}^{t} M_{s} \lambda_{s} d s\right|\right\} .
$$

Let $p \in(1,2)$ be a constant determined later, and let $q>2$ be its conjugate. Denote $M_{t}^{*} \triangleq \sup _{0 \leq s \leq t}\left|M_{s}\right|$ and $p_{1} \triangleq p\left(1+\frac{1}{q}\right)=2 p-1$. Applying the Burkholder-DavisGundy inequality we have

$$
\begin{aligned}
\tilde{E}\{\mid & \left.\int_{0}^{t} M_{s} \beta_{s} d \tilde{W}_{s}+\int_{0}^{t} M_{s} \lambda_{s} d s \mid\right\} \\
& \leq \tilde{E}\left\{\left(\int_{0}^{t}\left|M_{s} \beta_{s}\right|^{2} d s\right)^{\frac{1}{2}}\right\}+\tilde{E}\left\{\int_{0}^{t}\left|M_{s} \lambda_{s}\right| d s\right\} \\
& \leq \tilde{E}\left\{\left|M_{t}^{*}\right|^{1+\frac{1}{q}}\left[\left(\int_{0}^{t} M_{s}^{-\frac{2}{q}}\left|\beta_{s}\right|^{2} d s\right)^{\frac{1}{2}}+\int_{0}^{t} M_{s}^{-\frac{1}{q}}\left|\lambda_{s}\right| d s\right]\right\} \\
& \leq C \tilde{E}\left\{\left|M_{t}^{*}\right|^{p_{1}}\right\}^{\frac{1}{p}} \tilde{E}\left\{\left[\left(\int_{0}^{t} M_{s}^{-\frac{2}{q}}\left|\beta_{s}\right|^{2} d s\right)^{\frac{q}{2}}+\left(\int_{0}^{t} M_{s}^{-\frac{1}{q}}\left|\lambda_{s}\right| d s\right)^{q}\right]\right\}^{\frac{1}{q}} \\
& \leq C \tilde{E}\left\{\left|M_{t}^{*}\right|^{p_{1}}\right\}^{\frac{1}{p}} \tilde{E}\left\{\int_{0}^{t} M_{s}^{-1}\left[\left|\beta_{s}\right|^{q}+\left|\lambda_{s}\right|^{q}\right] d s\right\}^{\frac{1}{q}}\left[t^{1-\frac{2}{q}}+t^{1-\frac{1}{q}}\right] \\
& \leq C \tilde{E}\left\{\left|M_{t}^{*}\right|^{p_{1}}\right\}^{\frac{1}{p}} E\left\{\int_{0}^{t}\left[\left|\beta_{s}\right|^{q}+\left|\lambda_{s}\right|^{q}\right] d s\right\}^{\frac{1}{q}}\left[t^{1-\frac{2}{q}}+t^{1-\frac{1}{q}}\right] .
\end{aligned}
$$


Note that $\left|\alpha_{t}\right| \leq K_{1}$ and that $M_{t}=\exp \left(-\int_{0}^{t} \alpha_{s} d \tilde{W}_{s}-\frac{1}{2} \int_{0}^{t} \alpha_{s}^{2} d s\right)$ is a $\tilde{P}$-martingale. Then by Doob's inequality one gets

$$
\begin{aligned}
& \tilde{E}\left\{\left|M_{t}^{*}\right|^{p_{1}}\right\} \leq C \tilde{E}\left\{\left|M_{t}\right|^{p_{1}}\right\}=\tilde{E}\left\{\exp \left(-p_{1} \int_{0}^{t} \alpha_{s} d \tilde{W}_{s}-\frac{p_{1}}{2} \int_{0}^{t} \alpha_{s}^{2} d s\right)\right\} \\
& \leq C \tilde{E}\left\{\exp \left(-p_{1} \int_{0}^{t} \alpha_{s} d \tilde{W}_{s}-\frac{p_{1}^{2}}{2} \int_{0}^{t} \alpha_{s}^{2} d s+\frac{p_{1}^{2}-p_{1}}{2} K_{1}^{2} t\right)\right\} \\
& =C \exp \left(\frac{p_{1}^{2}-p_{1}}{2} K_{1}^{2} t\right),
\end{aligned}
$$

where the last equality, thanks to the fact that $\exp \left(-p_{1} \int_{0}^{t} \alpha_{s} d \tilde{W}_{s}-\frac{p_{1}^{2}}{2} \int_{0}^{t} \alpha_{s}^{2} d s\right)$, is a $\tilde{P}$-martingale. Plugging this into (3.19) and then into (3.18) we get

$$
\tilde{E}\left\{\mid N_{t} \Lambda_{t}^{-1}\right\} \leq|x|+K_{2} \int_{0}^{t} \tilde{E}\left\{\left|N_{s} \Lambda_{s}^{-1}\right|\right\} d s+C A_{t},
$$

where

$$
A_{t} \triangleq \exp \left(\frac{p_{1}^{2}-p_{1}}{2 p} K_{1}^{2} t\right) E\left\{\int_{0}^{t}\left[\left|\beta_{s}\right|^{q}+\left|\lambda_{s}\right|^{q}\right] d s\right\}^{\frac{1}{q}}\left[t^{1-\frac{2}{q}}+t^{1-\frac{1}{q}}\right] .
$$

Note that $A_{t}$ is increasing in $t$. The by the Gronwall inequality we have

$$
\tilde{E}\left\{\left|\Lambda_{t}^{-1} N_{t}\right|\right\} \leq C e^{K_{2} t}\left[|x|+A_{t}\right] .
$$

Now choose $p \triangleq 1+\frac{\varepsilon}{\varepsilon+6 K_{1}^{2}}$. One can easily check that $\frac{p_{1}^{2}-p_{1}}{2 p} K_{1}^{2} \leq \frac{\varepsilon}{2}$ and thus

$$
A_{t} \leq C e^{\varepsilon t} E\left\{\int_{0}^{t}\left[\left|\beta_{s}\right|^{q}+\left|\lambda_{s}\right|^{q}\right] d s\right\}^{\frac{1}{q}}
$$

which, combined with (3.17) and (3.20), proves (3.15) and hence the lemma.

Proof of Theorem 2.4. For any $x$, let $X$ and $X^{h}$ be defined as in (3.2) and (3.6), respectively. Denote $\Delta X_{t} \triangleq X_{t}-X_{t}^{h}$. Then

$$
\Delta X_{t}=\int_{0}^{t}\left[\alpha_{s} \Delta X_{s}+\beta_{s}\right] d W_{s}+\int_{0}^{t}\left[\gamma_{s} \Delta X_{s}+\lambda_{s}\right] d s,
$$

where

$$
\begin{cases}\alpha_{t} \triangleq \frac{\sigma\left(X_{t}\right)-\sigma\left(X_{t}^{h}\right)}{\Delta X_{t}}, & \beta_{t} \triangleq \sigma\left(X_{t}^{h}\right)-\sigma\left(X_{\tau(t)}^{h}\right), \\ \gamma_{t} \triangleq \frac{b\left(X_{t}\right)-b\left(X_{t}^{h}\right)}{\Delta X_{t}}, & \lambda_{t} \triangleq b\left(X_{t}^{h}\right)-b\left(X_{\tau(t)}^{h}\right) .\end{cases}
$$

By the Lipschitz continuity of $\sigma, b$, and recalling (3.5) we have

$$
\left|\alpha_{t}\right| \leq C, \quad\left|\gamma_{t}\right| \leq L_{b}, \quad\left|\beta_{t}\right|+\left|\lambda_{t}\right| \leq C h .
$$

Since $\beta L_{b}<1$, choose $\varepsilon \triangleq \frac{\beta^{-1}-L_{b}}{2}>0$. By (3.16) and the fact that $\Delta X_{0}=0$, we get

$$
E\left|\Delta X_{t}\right| \leq C h e^{\left(L_{b}+\varepsilon\right) t}
$$


Since $f$ is bounded and Hölder- $\alpha$ continuous, it is also Hölder- $\alpha \wedge \beta$ continuous. By (3.5) and (3.21) we have

$$
\begin{aligned}
& \left.E\left\{\left|f\left(X_{t}\right)-f\left(X_{\tau(t)}^{h}\right)\right|\right\} \leq\left. C E\left\{\mid X_{t}-X_{\tau(t)}^{h}\right)\right|^{\alpha \wedge \beta}\right\} \\
& \leq C E\left\{\left|\Delta X_{t}\right|^{\alpha \wedge \beta}+\left|X_{t}^{h}-X_{\tau(t)}^{h}\right|^{\alpha \wedge \beta}\right\} \leq C\left[\left(E\left\{\left|\Delta X_{t}\right|\right\}\right)^{\alpha \wedge \beta}+h^{\alpha \wedge \beta}\right] \\
& \leq C h^{\alpha \wedge \beta} e^{(\alpha \wedge \beta)\left(L_{b}+\varepsilon\right) t} .
\end{aligned}
$$

Then by (3.1) and (3.7) we get

$$
\begin{aligned}
& \left|u(x)-u_{h}^{w}(x)\right| \leq C \int_{0}^{\infty} e^{-t} E\left\{\left|f\left(X_{t}\right)-f\left(X_{\tau(t)}^{h}\right)\right|\right\} d t \\
& \leq C h^{\alpha \wedge \beta} \int_{0}^{\infty} e^{\left[(\alpha \wedge \beta)\left(L_{b}+\varepsilon\right)-1\right] t} d t=C h^{\alpha \wedge \beta},
\end{aligned}
$$

thanks to the fact that $\beta\left(L_{b}+\varepsilon\right)<1$. The proof for (2.10) is now complete.

\section{THE STANDARD FINITE DIFFERENCE SCHEME}

We prove Theorems 2.1 and 2.2 in this section, but again leave the sharpness to $\S 5$. The main idea is to transform (2.6) to the form of (2.8) and thus find the probability solution to (2.6). To be specific, in light of (2.7) and (2.9), we define two functions $\sigma_{h}(x)$ and $b_{h}(x)$ by the following equations:

$$
\left\{\begin{array}{l}
\exp \left(\frac{b_{h} h}{\sigma_{h}^{2}}\right)\left[\cosh \left(\frac{\sqrt{b_{h}^{2}+2 \sigma_{h}^{2}}}{\sigma_{h}^{2}} h\right)\right]^{-1}=\frac{\sigma^{2}+2 b h}{\sigma^{2}+b h+h^{2}}, \\
\exp \left(-\frac{b_{h} h}{\sigma_{h}^{2}}\right)\left[\cosh \left(\frac{\sqrt{b_{h}^{2}+2 \sigma_{h}^{2}}}{\sigma_{h}^{2}} h\right)\right]^{-1}=\frac{\sigma^{2}}{\sigma^{2}+b h+h^{2}} .
\end{array}\right.
$$

Now by the arguments in $\S 3$ we know that

$$
u_{h}^{s}(x)=E_{x}\left\{\int_{0}^{\infty} e^{-t} f\left(\tilde{X}_{\tilde{\tau}(t)}^{h}\right) d t\right\}
$$

and

$$
\left|u(x)-u_{h}^{s}(x)\right| \leq \int_{0}^{\infty} e^{-t}\left|E\left\{f\left(X_{t}\right)-f\left(\tilde{X}_{\tilde{\tau}(t)}^{h}\right)\right\}\right| d t,
$$

where $\tilde{\tau}$ and $\tilde{X}^{h}$ are defined in the same manner as (3.3) and (3.4), with $\sigma$ and $b$ replaced by $\sigma_{h}$ and $b_{h}$, respectively. Moreover, we have

$$
\left|\tilde{X}_{\tilde{\tau}(t)}^{h}-\tilde{X}_{t}^{h}\right| \leq h, \quad \text { a.s. }
$$

and

$$
\tilde{X}_{t}^{h}=x+\int_{0}^{t} \sigma_{h}\left(\tilde{X}_{\tilde{\tau}(s)}^{h}\right) d W_{s}+\int_{0}^{t} b_{h}\left(\tilde{X}_{\tilde{\tau}(s)}^{h}\right) d s .
$$

To prove the theorems, we need to estimate the errors of $\sigma_{h}, b_{h}$. Solving (4.1) directly we obtain

$$
\sigma_{h}=\frac{\sqrt{2} h}{\sqrt{\log \frac{r(\sigma, b, h)}{\sigma^{2}} \log \frac{r(\sigma, b, h)}{\sigma^{2}+2 b h}}}, \quad b_{h}=\frac{h \log \left(1+\frac{2 b h}{\sigma^{2}}\right)}{\log \frac{r(\sigma, b, h)}{\sigma^{2}} \log \frac{r(\sigma, b, h)}{\sigma^{2}+2 b h}},
$$

where

$$
r(\sigma, b, h) \triangleq \sigma^{2}+b h+h^{2}+h \sqrt{2 \sigma^{2}+b^{2}+2 b h+h^{2}} .
$$


When $\sigma=0$, we define $\sigma_{h}, b_{h}$ as the limits in (4.5). That is,

$$
\sigma_{h}=0, \quad b_{h}=\frac{h}{\log \left(1+\frac{h}{b}\right)} .
$$

We note that in general $\sigma_{h}, b_{h}$ are not Lipschitz continuous in $x$. Following are some key estimates whose proof is quite lengthy and thus postponed to $\S 6$.

Lemma 4.1. There exists a universal constant $C$ such that

$$
\left|\sigma_{h}-\sigma\right| \leq C\left[1+\frac{b}{\sqrt{\sigma^{2}+b h}}\right] h, \quad\left|b_{h}-b\right| \leq C h .
$$

Consequently we have

Corollary 4.2. (i) If $b$ is bounded, then $\left|\sigma_{h}-\sigma\right| \leq C \sqrt{h}$.

(ii) If $\frac{b}{\sigma}$ is bounded, then $\left|\sigma_{h}-\sigma\right| \leq C h$.

(iii) If both $\sigma$ and $b$ are bounded, then $\left|\sigma_{h}^{2}-\sigma^{2}\right| \leq C h$.

Proof. (i) and (ii) are direct consequence of Lemma 4.1 .

It remains to prove (iii). Since $b$ is bounded, by (i) we have $\left|\sigma_{h}\right| \leq|\sigma|+C \sqrt{h}$, and thus

$$
\begin{aligned}
& \left|\sigma_{h}^{2}-\sigma^{2}\right|=\left|\sigma_{h}-\sigma\right|\left|\sigma_{h}+\sigma\right| \leq C\left[1+\frac{b}{\sqrt{\sigma^{2}+b h}}\right] h[2|\sigma|+C \sqrt{h}] \\
& \leq C h\left[|\sigma|+\sqrt{h}+\frac{b|\sigma|+b \sqrt{h}}{\sqrt{\sigma^{2}+b h}}\right] \leq C h\left[1+\frac{|\sigma|+\sqrt{b h}}{\sqrt{\sigma^{2}+b h}}\right] \leq C h .
\end{aligned}
$$

That proves the lemma.

We note that all the conditions in Corollary 4.2 are necessary. Following are some counterexamples. All the calculations are straightforward, and thus we omit the proof.

Example 1. (i) ( $b$ is unbounded.)

Let $\sigma=1, b=\frac{1}{h}$. Then $\sigma_{h} \sim \sqrt{\frac{2}{\log 3}}$ and thus $\sigma_{h}-\sigma \sim \sqrt{\frac{2}{\log 3}}-1$.

(ii) ( $b$ is bounded, but $\frac{b}{\sigma}$ is unbounded.)

Let $\sigma=\sqrt{h}, b=1$, then $\sigma_{h} \sim \sqrt{\frac{2 h}{\log 3}}$, and thus $\sigma_{h}-\sigma \sim\left[\sqrt{\frac{2}{\log 3}}-1\right] \sqrt{h}$.

(iii) ( $b$ is bounded, but $\sigma$ is unbounded.)

Let $\sigma=\frac{1}{h}, b=1$; then $\sigma_{h}^{2} \sim \frac{1}{h^{2}}+\frac{1}{2}$, and thus $\sigma_{h}^{2}-\sigma^{2} \sim \frac{1}{2}$.

Proof of Theorem 2.2. Recall (4.3). Following the arguments in the proof of Theorem 2.4 we have

$$
\left|u(x)-u_{h}^{s}(x)\right| \leq C \int_{0}^{\infty} e^{-t} E\left\{\left|X_{t}-\tilde{X}_{t}^{h}\right|^{\alpha \wedge \beta}\right\} d t+C h^{\alpha \wedge \beta} .
$$

Denote $\Delta \tilde{X}_{t} \triangleq X_{t}-\tilde{X}_{t}^{h}$. Then

$$
\begin{aligned}
& d \Delta \tilde{X}_{t}=\left[\sigma\left(X_{t}\right)-\sigma_{h}\left(\tilde{X}_{\tilde{\tau}(t)}^{h}\right)\right] d W_{t}+\left[b\left(X_{t}\right)-b_{h}\left(\tilde{X}_{\tilde{\tau}(t)}^{h}\right)\right] d t \\
& =\left[\alpha_{t} \Delta \tilde{X}_{t}+\beta_{t}\right] d W_{t}+\left[\gamma_{t} \Delta \tilde{X}_{t}+\lambda_{t}\right] d t,
\end{aligned}
$$

where

$$
\begin{gathered}
\alpha_{t} \triangleq \frac{\sigma\left(X_{t}\right)-\sigma\left(\tilde{X}_{t}^{h}\right)}{\Delta \tilde{X}_{t}}, \quad \beta_{t} \triangleq \sigma\left(\tilde{X}_{t}^{h}\right)-\sigma_{h}\left(\tilde{X}_{\tilde{\tau}(t)}^{h}\right), \\
\gamma_{t} \triangleq \frac{b\left(X_{t}\right)-b\left(\tilde{X}_{t}^{h}\right)}{\Delta \tilde{X}_{t}}, \quad \lambda_{t} \triangleq b\left(\tilde{X}_{t}^{h}\right)-b_{h}\left(\tilde{X}_{\tilde{\tau}(t)}^{h}\right) .
\end{gathered}
$$


Since $\sigma$ and $b$ are Lipschitz continuous, we have $\left|\alpha_{t}\right| \leq C$ and $\left|\gamma_{t}\right| \leq L_{b}$. Moreover, by Corollary 4.2 (i) we have $\left|\sigma-\sigma_{h}\right| \leq C \sqrt{h}$, therefore,

$$
\left|\beta_{t}\right| \leq\left|\sigma\left(\tilde{X}_{t}^{h}\right)-\sigma\left(\tilde{X}_{\tilde{\tau}(t)}^{h}\right)\right|+\left|\sigma\left(\tilde{X}_{\tilde{\tau}(t)}^{h}\right)-\sigma_{h}\left(\tilde{X}_{\tilde{\tau}(t)}^{h}\right)\right| \leq C \sqrt{h}
$$

Similarly, by Lemma 4.1 we have $\left|\lambda_{t}\right| \leq C h$. Now letting $0<\varepsilon<\beta^{-1}-L_{b}$ and applying Lemma 3.2, we get $E\left\{\left|\Delta \tilde{X}_{t}\right|\right\} \leq C \sqrt{h} e^{\left(L_{b}+\varepsilon\right) t}$, which, combined with (4.7), proves (i).

It remains to prove (ii). We note that if $\sigma=0$, by (4.6) we have $\left|\sigma_{h}-\sigma\right|=0 \leq$ $C h$. If $\frac{b}{\sigma}$ is bounded, by Corollary 4.2 (ii) we have $\left|\sigma_{h}-\sigma\right| \leq C h$. Now (ii) follows a line-by-line analogy as above.

We next prove Theorem 2.1, Recall (4.3). Note that we estimate the error of $f\left(\tilde{X}_{\tilde{\tau}(t)}^{h}\right)-f\left(X_{t}\right)$ in (4.7) in the strong sense. The next lemma gives an estimate of the error in the weak sense. The idea is more or less standard (see, e.g., [22]).

Lemma 4.3. Assume all the conditions in Theorem 2.1 hold true. Then

$$
\left|E\left\{f\left(\tilde{X}_{T}^{h}\right)-f\left(X_{T}\right)\right\}\right| \leq C e^{\frac{1+L}{2} T} h, \quad \forall T>0 .
$$

Proof. Fix $T>0$. Let $v$ be the solution to the following degenerate parabolic PDE:

$$
\left\{\begin{array}{l}
v_{t}+\frac{1}{2} \sigma^{2}(x) v_{x x}+b(x) v_{x}=0, \quad t \in(0, T), \\
v(T, x)=f(x)
\end{array}\right.
$$

Then we have

$$
E\left\{f\left(\tilde{X}_{T}^{h}\right)\right\}=E\left\{v\left(T, \tilde{X}_{T}^{h}\right)\right\}, \quad E\left\{f\left(X_{T}\right)\right\}=v(0, x) .
$$

Obviously $v \in C^{1,2}$. Applying the Ito formula one has

$$
\begin{aligned}
d v\left(t, \tilde{X}_{t}^{h}\right) & \\
= & {\left[v_{t}+\frac{1}{2} \sigma_{h}^{2}\left(\tilde{X}_{\tilde{\tau}(t)}^{h}\right) v_{x x}+b_{h}\left(\tilde{X}_{\tilde{\tau}(t)}^{h}\right) v_{x}\right]\left(t, \tilde{X}_{t}^{h}\right) d t+\sigma_{h}^{2}\left(\tilde{X}_{\tilde{\tau}(t)}^{h}\right) v_{x}\left(t, \tilde{X}_{t}^{h}\right) d W_{t} } \\
= & {\left[\frac{1}{2}\left[\sigma_{h}^{2}\left(\tilde{X}_{\tilde{\tau}(t)}^{h}\right)-\sigma^{2}\left(\tilde{X}_{t}^{h}\right)\right] v_{x x}+\left[b_{h}\left(\tilde{X}_{\tilde{\tau}(t)}^{h}\right)-b\left(\tilde{X}_{t}^{h}\right)\right] v_{x}\right]\left(t, \tilde{X}_{t}^{h}\right) d t } \\
& +\sigma_{h}\left(\tilde{X}_{\tilde{\tau}(t)}^{h}\right) v_{x}\left(t, \tilde{X}_{t}^{h}\right) d W_{t} .
\end{aligned}
$$

By (4.4), Corollary 4.2(iii) and Lemma 4.1 we have

$$
\begin{aligned}
& \left|\sigma_{h}^{2}\left(\tilde{X}_{\tilde{\tau}(t)}^{h}\right)-\sigma^{2}\left(\tilde{X}_{t}^{h}\right)\right| \leq\left|\sigma_{h}^{2}\left(\tilde{X}_{\tilde{\tau}(t)}^{h}\right)-\sigma^{2}\left(\tilde{X}_{\tilde{\tau}(t)}^{h}\right)\right|+\left|\sigma^{2}\left(\tilde{X}_{\tilde{\tau}(t)}^{h}\right)-\sigma^{2}\left(\tilde{X}_{t}^{h}\right)\right| \leq C h, \\
& \left|b_{h}\left(\tilde{X}_{\tilde{\tau}(t)}^{h}\right)-b\left(\tilde{X}_{t}^{h}\right)\right| \leq\left|b_{h}\left(\tilde{X}_{\tilde{\tau}(t)}^{h}\right)-b\left(\tilde{X}_{\tilde{\tau}(t)}^{h}\right)\right|+\left|b\left(\tilde{X}_{\tilde{\tau}(t)}^{h}\right)-b\left(\tilde{X}_{t}^{h}\right)\right| \leq C h .
\end{aligned}
$$

We claim that

$$
\left|v_{x}(t, x)\right|+\left|v_{x x}(t, x)\right| \leq C e^{\frac{1+L}{2}(T-t)}, \quad \forall t \in[0, T] .
$$

Then

$$
\left|E\left\{v\left(T, \tilde{X}_{T}^{h}\right)-v(0, x)\right\}\right| \leq C h \int_{0}^{T} e^{\frac{1+L}{2}(T-t)} d t \leq C e^{\frac{1+L}{2} T} h,
$$

which, together with (4.9), proves the lemma.

It remains to prove (4.10). Without loss of generality, we shall prove it only at $t=0$. To this end, we recall (4.9). Let $\nabla X, \nabla^{2} X$ denote the first order and second 
order derivative flows of $X$, differentiating with respect to $x$. That is,

$$
\begin{aligned}
\nabla X_{t}= & 1+\int_{0}^{t} \sigma^{\prime}\left(X_{s}\right) \nabla X_{s} d W_{s}+\int_{0}^{t} b^{\prime}\left(X_{s}\right) \nabla X_{s} d s \\
\nabla^{2} X_{t}= & \int_{0}^{t}\left[\sigma^{\prime}\left(X_{s}\right) \nabla^{2} X_{s}+\sigma^{\prime \prime}\left(X_{s}\right)\left|\nabla X_{s}\right|^{2}\right] d W_{s} \\
& +\int_{0}^{t}\left[b^{\prime}\left(X_{s}\right) \nabla^{2} X_{s}+b^{\prime \prime}\left(X_{s}\right)\left|\nabla X_{s}\right|^{2}\right] d s .
\end{aligned}
$$

Then we have

$$
v_{x}(0, x)=E\left\{f^{\prime}\left(X_{T}\right) \nabla X_{T}\right\}, \quad v_{x x}(0, x)=E\left\{f^{\prime}\left(X_{T}\right) \nabla^{2} X_{T}+f^{\prime \prime}\left(X_{T}\right)\left|\nabla X_{T}\right|^{2}\right\} .
$$

For $\varepsilon \triangleq \frac{1-L}{2}>0$, applying (3.16) to (4.11) we get

$$
E\left\{\left|\nabla X_{T}\right|\right\} \leq C e^{\left(L_{b}+\varepsilon\right) T} \leq C e^{\frac{1+L}{2} T} .
$$

Moreover, for any $q>2$, applying the Ito formula we have

$$
\left|\nabla X_{t}\right|^{q}=1+\int_{0}^{t} q \sigma^{\prime}\left(X_{s}\right)\left|\nabla X_{s}\right|^{q} d W_{s}+\int_{0}^{t}\left[q b^{\prime}\left(X_{s}\right)+\frac{q(q-1)}{2}\left|\sigma^{\prime}\left(X_{s}\right)\right|^{2}\right]\left|\nabla X_{s}\right|^{q} d s .
$$

Then letting $q$ be close to 2 enough and applying (3.16) again one gets

$$
E\left\{\left|\nabla X_{T}\right|^{q}\right\} \leq C e^{\frac{1+L}{2} T} .
$$

Now applying (3.15) to (4.12) we have

$$
E\left\{\left|\nabla^{2} X_{T}\right|\right\} \leq C e^{\frac{1+L}{2} T} .
$$

Now by (4.14), (4.15), (4.16), and (4.13) we prove (4.10), hence the lemma.

Proof of Theorem 2.1. Recalling (4.3) and (4.4), and applying Lemma 4.3 we have

$$
\begin{aligned}
& \left|u(x)-u_{h}^{s}(x)\right| \leq C \int_{0}^{\infty} e^{-t}\left|E\left\{\left[f\left(\tilde{X}_{\tilde{t}(t)}^{h}\right)-f\left(\tilde{X}_{t}^{h}\right)\right]+\left[f\left(\tilde{X}_{t}^{h}\right)-f\left(X_{t}\right)\right]\right\}\right| d t \\
& \leq C \int_{0}^{\infty} e^{-t}\left[h+e^{\frac{1+L}{2} t} h\right] d t=C h \int_{0}^{\infty}\left[e^{-t}+e^{\frac{L-1}{2} t}\right] d t=C h,
\end{aligned}
$$

thanks to the assumption that $L<1$.

\section{Sharpness of the estimates}

In this section we give three examples to show that some estimates in $\S 2$ are sharp. We first consider $\beta$.

Example 2. Let $\sigma=0, b(x)=L_{b} x, f(x)=0$ for $x \in[0,1]$. Choose $\sigma, b, f \in C_{0}^{\infty}$ appropriately outside $[0,1]$ such that $u(1)=1$. Then, by letting $h=\frac{1}{n}$,

$$
\liminf _{n \rightarrow \infty} n^{\frac{1}{L_{b}}} \sup _{x \in[0,1]}\left|u(x)-u_{h}^{s}(x)\right|>0, \quad \liminf _{n \rightarrow \infty} n^{\frac{1}{L_{b}}} \sup _{x \in[0,1]}\left|u(x)-u_{h}^{w}(x)\right|>0 .
$$

Proof. We will only estimate $u(x)-u_{h}^{w}(x)$. The other one is similar.

First, under our assumptions on $[0,1]$ (1.1) becomes $L_{b} x u^{\prime}(x)-u(x)=0$. Since $u(1)=1$, one has

$$
u(x)=x^{\frac{1}{L_{b}}}, \forall x \in[0,1] .
$$


On the other hand, for $h=\frac{1}{n}$, and $k=1, \cdots, n-1$, by (2.8) we have $u_{h}^{w}(k h)=$ $\exp \left(-\frac{1}{L_{b} k}\right) u_{h}^{w}((k+1) h)$, which leads to

$$
u_{h}^{w}(h)=u_{h}^{w}(1) \exp \left(-\frac{1}{L_{b}} \sum_{k=1}^{n-1} \frac{1}{k}\right) .
$$

If $\left|u_{h}^{w}(1)-u(1)\right| \geq h^{\frac{1}{L_{b}}}$, then we have

$$
n^{\frac{1}{L_{b}}}\left|u_{h}^{w}(1)-u(1)\right| \geq 1 .
$$

Now we assume $\left|u_{h}^{w}(1)-1\right|=\left|u_{h}^{w}(1)-u(1)\right|<h^{\frac{1}{L_{b}}}$. Note that

$$
\sum_{k=1}^{n-1} \frac{1}{k} \geq 1+\int_{2}^{n} \frac{d x}{x}=1-\log (2)+\log (n)
$$

By (5.2) we have

$u_{h}^{w}(h) \leq\left(1+h^{\frac{1}{L_{b}}}\right) \exp \left(-\frac{1}{L_{b}}[1-\log (2)+\log (n)]\right)=\left(1+h^{\frac{1}{L_{b}}}\right) \exp \left(-\frac{1-\log (2)}{L_{b}}\right) h^{\frac{1}{L_{b}}}$,

which, combined with (5.1), implies that

$$
u(h)-u_{h}^{w}(h) \geq\left[1-\left(1+h^{\frac{1}{L_{b}}}\right) \exp \left(-\frac{1-\log (2)}{2 L_{b}}\right)\right] h^{\frac{1}{L_{b}}} .
$$

Note that $1-\log (2)>0$. When $h$ is small enough, we have

$$
\left(1+h^{\frac{1}{L_{b}}}\right) \exp \left(-\frac{1-\log (2)}{L_{b}}\right) \leq \exp \left(-\frac{1-\log (2)}{2 L_{b}}\right)<1 .
$$

Then

$$
n^{\frac{1}{L_{b}}}\left[u(h)-u_{h}^{w}(h)\right] \geq 1-\exp \left(-\frac{1-\log (2)}{2 L_{b}}\right)>0,
$$

which, combined with (5.3), proves our claim.

The next example shows that the first order estimate in (2.3) is sharp.

Example 3. Let $\sigma(x)=\sqrt{h}, b(x)=1, f(x)=0$ for $x \in[0,1]$. Choose $\sigma, b, f \in C_{0}^{\infty}$ appropriately outside $[0,1]$ such that $u(0)=0$ and $u(1)=1$. Then, by letting $h=\frac{1}{n}$,

$$
\liminf _{n \rightarrow \infty} n \sup _{x \in[0,1]}\left|u(x)-u_{h}^{s}(x)\right|>0 .
$$

We note that in this example $\sigma$, and thus $u$, depends on $h$. But one may divide $\mathbb{R}$ into countable disjoint intervals $\left[a_{n}, b_{n}\right)$. By Example 3 we can set $\sigma, b, f$ on each interval such that they all vanish on $a_{n}, b_{n}$ and $n \sup _{x \in\left[a_{n}, b_{n}\right]}\left|u(x)-u_{\frac{1}{n}}^{s}(x)\right| \geq c_{0}$ for some constant $c_{0}>0$ which is independent of $n$. Combining all the pieces together we get universal $\sigma, b, f$ satisfying our requirement.

Proof of Example 3. First, one can easily solve (1.1):

$$
u(x)=\frac{e^{\lambda_{1} x}-e^{\lambda_{2} x}}{e^{\lambda_{1}}-e^{\lambda_{2}}}, \quad x \in[0,1],
$$

where

$$
\lambda_{1} \triangleq \frac{-1+\sqrt{1+2 h}}{h}, \quad \lambda_{2} \triangleq \frac{-1-\sqrt{1+2 h}}{h} .
$$


On the other hand, solve (2.6) we have

$$
u_{h}^{s}(k h)=c_{1} \gamma_{1}^{k}-c_{2} \gamma_{2}^{k}, \quad k=0,1 \cdots, n,
$$

for some constants $c_{1}, c_{2}$, where

$$
\gamma_{1} \triangleq \frac{2+h+\sqrt{1+4 h+h^{2}}}{3}, \quad \gamma_{2} \triangleq \frac{2+h-\sqrt{1+4 h+h^{2}}}{3} .
$$

If $\left|u_{h}^{s}(0)\right| \geq \varepsilon h$ or $\left|u_{h}^{s}(1)-1\right| \geq \varepsilon h$ where $\varepsilon>0$ is a small number independent of $h$ which will be determined later, then we have

$$
n \sup _{x \in[0,1]}\left|u(x)-u_{h}^{s}(x)\right| \geq \varepsilon .
$$

Now we assume $\left|u_{h}^{s}(0)\right| \leq \varepsilon h$ and $\left|u_{h}^{s}(1)-1\right| \leq \varepsilon h$. By letting $k=0$ and $k=n$ in (5.4) we get

Denote

$$
c_{1}=\frac{u_{h}^{s}(1)-u_{h}^{s}(0) \gamma_{2}^{n}}{\gamma_{1}^{n}-\gamma_{2}^{n}}, \quad c_{2}=\frac{u_{h}^{s}(1)-u_{h}^{s}(0) \gamma_{1}^{n}}{\gamma_{1}^{n}-\gamma_{2}^{n}} .
$$

$$
\bar{u}_{h}^{s}(k h) \triangleq \frac{\gamma_{1}^{k}-\gamma_{2}^{k}}{\gamma_{1}^{n}-\gamma_{2}^{n}}, \quad k=0,1 \cdots, n .
$$

Note that for $\gamma_{1}>1>\frac{1}{3}>\gamma_{2}>0$ and $\gamma_{1} \gamma_{2}=\frac{1}{3}$, we have

$$
\left|\bar{u}_{h}^{s}(k h)-u_{h}^{s}(k h)\right|=\left|\frac{\left[u_{h}^{s}(1)-1\right]\left[\gamma_{1}^{k}-\gamma_{2}^{k}\right]-u_{h}^{s}(0)\left[\gamma_{1}^{k} \gamma_{2}^{n}-\gamma_{1}^{n} \gamma_{2}^{k}\right]}{\gamma_{1}^{n}-\gamma_{2}^{n}}\right| \leq 2 \varepsilon h .
$$

Now we estimate $\left|u(k h)-\bar{u}_{h}^{s}(k h)\right|$. Let $x=\frac{1}{2}$, or say, $k=\frac{n}{2}$. Then we have

$$
u\left(\frac{1}{2}\right)=\left[e^{\frac{\lambda_{1}}{2}}+e^{\frac{\lambda_{2}}{2}}\right]^{-1} \sim e^{-\frac{\lambda_{1}}{2}}, \quad \bar{u}_{h}^{s}\left(\frac{1}{2}\right)=\left[\gamma_{1}^{\frac{n}{2}}+\gamma_{2}^{\frac{n}{2}}\right]^{-1} \sim \exp \left(-\frac{n}{2} \log \left(\gamma_{1}\right)\right) .
$$

Note that $\frac{\lambda_{1}}{2} \sim \frac{1}{2}-\frac{h}{4}$, and

$$
\frac{n}{2} \log \left(\gamma_{1}\right) \sim \frac{1}{2 h} \log \left(1+h-\frac{h^{2}}{2}\right) \sim \frac{1}{2 h}\left(h-h^{2}\right)=\frac{1}{2}-\frac{h}{2} .
$$

Then,

$$
u\left(\frac{1}{2}\right)-\bar{u}_{h}^{s}\left(\frac{1}{2}\right) \sim e^{-\frac{1}{2}+\frac{h}{4}}-e^{-\frac{1}{2}+\frac{h}{2}} \sim-\frac{1}{4} e^{-\frac{1}{2}} h .
$$

Now let $\varepsilon \triangleq \frac{1}{15} e^{-\frac{1}{2}}>0$. When $h$ is small enough, by (5.6) we have

$$
\left|u\left(\frac{1}{2}\right)-u_{h}^{s}\left(\frac{1}{2}\right)\right| \geq\left|u\left(\frac{1}{2}\right)-u_{h}^{s}\left(\frac{1}{2}\right)\right|-\left|u_{h}^{s}\left(\frac{1}{2}\right)-\bar{u}_{h}^{s}\left(\frac{1}{2}\right)\right| \geq \frac{1}{5} e^{-\frac{1}{2}} h-2 \varepsilon h \geq \varepsilon h,
$$

which, combined with (5.5), proves our claim.

Our last example shows the sharpness of $\alpha$. Again we will only prove it for $u_{h}^{w}$.

Example 4. Assume $\alpha \in(0,1]$. Let $\sigma \triangleq \sqrt{2}, b \triangleq 0$, and $f(x) \triangleq \sum_{m=0}^{\infty} f_{m}(x)$, where $f_{m}$ has period $2^{-m}$, and

$$
f_{m}(x) \triangleq \begin{cases}x^{\alpha}, & 0 \leq x<2^{-(m+1)}, \\ \left(2^{-m}-x\right)^{\alpha}, & 2^{-(m+1)} \leq x<2^{-m} .\end{cases}
$$

Then

(i) $f$ is bounded;

(ii) $|f(x)-f(y)| \leq C|x-y|^{\alpha} \log \frac{1}{|x-y|}, \quad \forall|x-y| \leq \frac{1}{2}$; 
(iii) for $h=2^{-n}$,

$$
\liminf _{n \rightarrow \infty} 2^{n \alpha}\left|u(0)-u_{h}^{w}(0)\right|>0
$$

Proof. (i) By (5.7) it obviously holds that $\left|f_{m}(x)\right| \leq 2^{-(m+1) \alpha}$, thus

$$
|f(x)| \leq \sum_{m=0}^{\infty} 2^{-(m+1) \alpha}=\frac{1}{2^{\alpha}-1}<\infty .
$$

(ii) For any $|x-y| \leq \frac{1}{2}$, assuming $2^{-n} \leq|x-y|<2^{1-n}$, we have

$$
\left|f_{m}(x)-f_{m}(y)\right| \leq \begin{cases}|x-y|^{\alpha}, & m<n, \\ 2^{1-(m+1) \alpha}, & m \geq n .\end{cases}
$$

Therefore,

$$
\begin{aligned}
& |f(x)-f(y)| \leq \sum_{m=0}^{\infty}\left|f_{m}(x)-f_{m}(y)\right| \leq \sum_{m=0}^{n-1}|x-y|^{\alpha}+\sum_{m=n}^{\infty} 2^{1-(m+1) \alpha} \\
& =n|x-y|^{\alpha}+2^{1-\alpha-n \alpha} \frac{1}{1-2^{-\alpha}} \leq C|x-y|^{\alpha} \log \frac{1}{|x-y|} .
\end{aligned}
$$

(iii) We first note that, in our example, (1.1) and (2.8) become

$$
u "-u=-f
$$

and

$$
u_{h}^{w}(x)=\frac{1}{2 \cosh (h)}\left[u_{h}^{w}(x+h)+u_{h}^{w}(x-h)\right]+\left(1-\frac{1}{\cosh (h)}\right) f(x),
$$

respectively. The general solution to (5.9) is

$$
u(x)=C_{1} e^{x}+C_{2} e^{-x}-\frac{1}{2} \int_{0}^{x}\left[e^{x-y}-e^{y-x}\right] f(y) d y .
$$

Note that, for bounded $f$, we require $u$ to be bounded. By letting $x \rightarrow \infty$ or $x \rightarrow-\infty$ we get

$$
C_{1}=\frac{1}{2} \int_{0}^{\infty} e^{-y} f(y) d y, \quad C_{2}=\int_{-\infty}^{0} e^{y} f(y) d y .
$$

Thus

$$
u(x)=\frac{1}{2} \int_{-\infty}^{\infty} e^{-|x-y|} f(y) d y .
$$

Moreover, noting that $f$ is even and $f(0)=0$, we get

$$
u(0)=\int_{0}^{\infty} e^{-x} f(x) d x .
$$

Analogously, the general solution to (5.10) is

$$
u_{h}^{w}(m h)=C_{1} e^{m h}+C_{2} e^{-m h}-\frac{\cosh (h)-1}{\sinh (h)} \sum_{k=0}^{m}\left[e^{(m-k) h}-e^{(k-m) h}\right] f(k h) .
$$

By similar arguments we get

$$
u_{h}^{w}(0)=\frac{2(\cosh (h)-1)}{\sinh (h)} \sum_{k=1}^{\infty} e^{-k h} f(k h) .
$$


We shall use (5.11) and (5.12) to prove (5.8). The proof is quite lengthy and purely analytic. We thus postpone it to the Appendix.

\section{Appendix}

In this Appendix we prove (5.8) and Lemma 4.1. All the arguments are purely analytic.

Proof of (5.8). We shall use (5.11) and (5.12) to estimate $\left|u(0)-u_{h}(0)\right|$. First,

$$
\begin{aligned}
& \int_{0}^{\infty} e^{-x} f_{m}(x) d x=\sum_{k=0}^{\infty} \int_{k 2^{-m}}^{(k+1) 2^{-m}} e^{-x} f_{m}(x) d x=\sum_{k=0}^{\infty} e^{-k 2^{-m}} \int_{0}^{2^{-m}} e^{-x} f_{m}(x) d x \\
& =\frac{1}{1-e^{-2^{-m}}}\left[\int_{0}^{2^{-(m+1)}} x^{\alpha} e^{-x} d x+\int_{2^{-(m+1)}}^{2^{-m}}\left(2^{-m}-x\right)^{\alpha} e^{-x} d x\right] \\
& =\frac{1}{1-e^{-2^{-m}}} \int_{0}^{2^{-(m+1)}} x^{\alpha}\left[e^{-x}+e^{x-2^{-m}}\right] d x .
\end{aligned}
$$

By (5.11) we have

$$
\begin{aligned}
u(0) & =\sum_{m=0}^{n-1} \frac{1}{1-e^{-2^{-m}}} \int_{0}^{2^{-(m+1)}} x^{\alpha}\left[e^{-x}+e^{x-2^{-m}}\right] d x \\
& +\sum_{m=n}^{\infty} \frac{1}{1-e^{-2^{-m}}} \int_{0}^{2^{-(m+1)}} x^{\alpha}\left[e^{-x}+e^{x-2^{-m}}\right] d x .
\end{aligned}
$$

Using substitution $m=n-m^{\prime}-1$ in the first summation and $m=n+m^{\prime}$ in the second summation, we get

$$
\begin{aligned}
u(0) & =\sum_{m=0}^{n-1} \frac{1}{1-e^{-2^{m-n+1}}} \int_{0}^{2^{m-n}} x^{\alpha}\left[e^{-x}+e^{x-2^{m-n+1}}\right] d x \\
& +\sum_{m=0}^{\infty} \frac{1}{1-e^{-2^{-m-n}}} \int_{0}^{2^{-(m+n+1)}} x^{\alpha}\left[e^{-x}+e^{x-2^{-m-n}}\right] d x .
\end{aligned}
$$

Then by letting $x=h x^{\prime}=2^{-n} x^{\prime}$, we have

$$
\begin{aligned}
u(0) & =h^{\alpha} \sum_{m=0}^{n-1} \frac{h}{1-e^{-2^{m+1} h}} \int_{0}^{2^{m}} x^{\alpha}\left[e^{-x h}+e^{x h-2^{m+1} h}\right] d x \\
& +h^{\alpha} \sum_{m=0}^{\infty} \frac{h}{1-e^{-2^{-m} h}} \int_{0}^{2^{-(m+1)}} x^{\alpha}\left[e^{-x h}+e^{x h-2^{-m}}\right] d x
\end{aligned}
$$


On the other hand, note that $f_{m}(k h)=0$ for $m \geq n$ and $f_{m}$ is periodic with period $2^{-m}$. So by (5.12) we have

$$
\begin{aligned}
u_{h}^{w}(0)= & \frac{2(\cosh (h)-1)}{\sinh (h)} \sum_{k=1}^{\infty} e^{-k h} \sum_{m=0}^{n-1} f_{m}(k h) \\
= & \frac{2(\cosh (h)-1)}{\sinh (h)} \sum_{m=0}^{n-1} \sum_{k=1}^{2^{n-m}-1} e^{-k h} f_{m}(k h) \sum_{l=0}^{\infty} e^{-2^{n-m} l h} \\
= & \frac{2(\cosh (h)-1)}{\sinh (h)} \sum_{m=0}^{n-1} \frac{1}{1-e^{-2^{n-m} h}} \\
& \times\left[\sum_{k=1}^{2^{n-m-1}-1}|k h|^{\alpha}\left(e^{-k h}+e^{\left(k-2^{n-m}\right) h}\right)+2^{-(m+1) \alpha} e^{-2^{n-m-1} h}\right] .
\end{aligned}
$$

Using the substitution $m=n-m^{\prime}-1$ again we have

$$
\begin{aligned}
u_{h}^{w}(0)= & h^{\alpha} \frac{2(\cosh (h)-1)}{\sinh (h)} \sum_{m=0}^{n-1} \frac{1}{1-e^{-2^{m+1} h}} \\
& \times\left[\sum_{k=1}^{2^{m}-1} k^{\alpha}\left(e^{-k h}+e^{\left(k-2^{m+1}\right) h}\right)+2^{m \alpha} e^{-2^{m} h}\right] .
\end{aligned}
$$

Subtracting (6.1) from (6.2), and dividing by $h^{\alpha}=2^{-n \alpha}$, we get

$$
\begin{aligned}
& 2^{n \alpha}\left[u_{h}^{w}(0)-u(0)\right] \\
&=-\sum_{m=0}^{\infty} \frac{h}{1-e^{-2^{-m} h}} \int_{0}^{2^{-(m+1)}} x^{\alpha}\left[e^{-x h}+e^{x h-2^{-m} h}\right] d x \\
&+\sum_{m=0}^{n-1}\left\{\frac{2(\cosh (h)-1)}{\sinh (h)\left(1-e^{-2^{m+1} h}\right)}\right. \\
& \times\left[\sum_{k=1}^{2^{m}-1} k^{\alpha}\left(e^{-k h}+e^{\left(k-2^{m+1}\right) h}\right)+2^{m \alpha} e^{-2^{m} h}\right] \\
&\left.-\frac{h}{1-e^{-2^{m+1} h}} \int_{0}^{2^{m}} x^{\alpha}\left[e^{-x h}+e^{x h-2^{m+1} h}\right] d x\right\} \\
&=-I(h)+\sum_{m=0}^{n-1} I_{m}(h),
\end{aligned}
$$

where $I$ and $I_{m}$ are defined in an obvious way. Now let $n \rightarrow \infty$, or equivalently, $h \rightarrow 0$. We shall study the limits of the terms in (6.3).

First,

$$
\begin{aligned}
& \frac{h}{1-e^{-2^{-m} h}} \int_{0}^{2^{-(m+1)}} x^{\alpha}\left[e^{-x h}+e^{x h-2^{-m} h}\right] d x \\
& \leq \frac{1}{1-e^{-2^{-m} h}} 2^{-(m+1) \alpha}\left[\left(1-e^{-2^{-(m+1)} h}\right)+e^{-2^{-m} h}\left(e^{2^{-(m+1)} h}-1\right)\right]=2^{-(m+1) \alpha} .
\end{aligned}
$$


Since $\sum_{m=1}^{\infty} 2^{-(m+1) \alpha}<\infty$, by the Dominated Convergence Theorem one gets that

$$
\begin{aligned}
\lim _{h \rightarrow 0} I(h) & =\sum_{m=0}^{\infty} \lim _{h \rightarrow 0} \frac{h}{1-e^{-2^{-m} h}} \int_{0}^{2^{-(m+1)}} x^{\alpha}\left[e^{-x h}+e^{x h-2^{-m} h}\right] d x \\
& =\sum_{m=0}^{\infty} 2^{m} \int_{0}^{2^{-(m+1)}} x^{\alpha} 2 d x \\
& =\sum_{m=0}^{\infty} \frac{1}{(1+\alpha) 2^{(m+1) \alpha}}=\frac{1}{(1+\alpha)\left(2^{\alpha}-1\right)} \triangleq I .
\end{aligned}
$$

To estimate $I_{m}(h)$, we discuss two cases. First, if $\alpha=1$, one can check directly that

$$
\sum_{k=1}^{2^{m}-1} k x^{k}=\frac{x}{(1-x)^{2}}\left(1-x^{2^{m}}\right)-\frac{x}{1-x} 2^{m} x^{2^{m}-1},
$$

and that

$$
\int_{0}^{2^{m}} x e^{a x} d x=\left[a^{-1} 2^{m}-a^{-2}\right] e^{2^{m} a}+a^{-2}
$$

Then by straightforward calculation we have

$$
I_{m}(h)=\left[\frac{1}{\sinh (h)}-\frac{1}{h}\right] \frac{1-e^{-2^{m} h}}{1+e^{-2^{m} h}} \leq 0 .
$$

Thus

$$
\limsup _{h \rightarrow 0} 2^{n}\left[u_{h}^{w}(0)-u(0)\right] \leq-I<0,
$$

which obviously proves (5.8) in the case that $\alpha=1$.

Now we assume $\alpha \in(0,1)$. Note that

$$
I_{m}(h)=I_{m}^{1}(h)+I_{m}^{2}(h)
$$

where

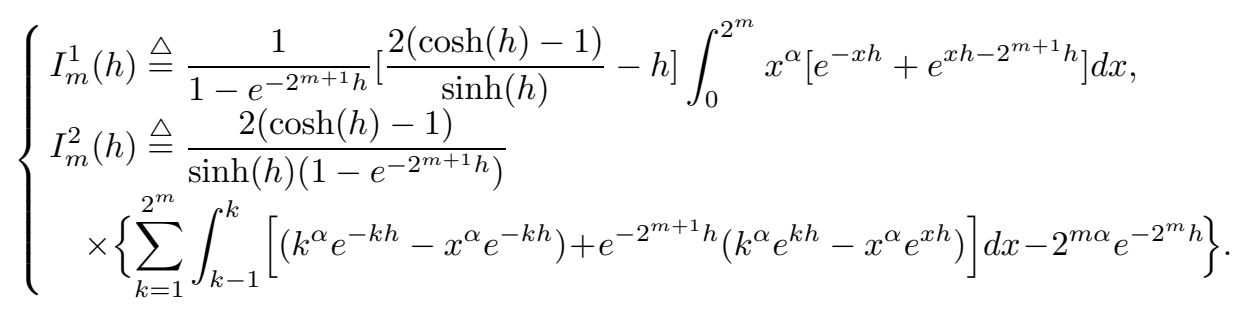

Now for $m<n$, we have

$$
\left|I_{m}^{1}(h)\right| \leq \frac{C h^{2}}{1-e^{-2^{m+1} h}} 2^{m \alpha} \int_{0}^{2^{m}}\left[e^{-x h}+e^{x h-2^{m+1} h}\right] d x=C h 2^{m \alpha} \leq C 2^{m(\alpha-1)} .
$$


Moreover, noting that

$$
|g(k)-g(x)| \leq \int_{x}^{k}\left|g^{\prime}(y)\right| d y
$$

for any differentiable function $g$ and any $x \in[k-1, k]$, we have

$$
\begin{aligned}
\left(1-e^{-2^{m+1} h}\right)\left|I_{m}^{2}(h)\right| & \\
\leq & C h\left\{\sum _ { k = 1 } ^ { 2 ^ { m } } \int _ { k - 1 } ^ { k } \left[\left|k^{\alpha} e^{-k h}-x^{\alpha} e^{-x h}\right|\right.\right. \\
& \left.\left.+e^{-2^{m+1} h}\left|k^{\alpha} e^{k h}-x^{\alpha} e^{x h}\right|\right] d x+2^{m \alpha} e^{-2^{m} h}\right\} \\
\leq & C h \sum_{k=1}^{2^{m}} \int_{k-1}^{k}\left[\left(\alpha x^{\alpha-1} e^{-x h}+h x^{\alpha} e^{-x h}\right)\right. \\
& \left.+e^{-2^{m+1} h}\left(\alpha x^{\alpha-1} e^{x h}+h x^{\alpha} e^{x h}\right)\right] d x+C h 2^{m \alpha} \\
\leq & C \alpha h \int_{0}^{2^{m}} x^{\alpha-1} d x \\
& +C h^{2} 2^{m \alpha} \int_{0}^{2^{m}}\left[e^{-x h}+e^{\left(x-2^{m+1}\right) h}\right] d x+C h 2^{m \alpha} \\
= & C h 2^{m \alpha}+C h 2^{m \alpha}\left[1-e^{-2^{m+1} h}\right]+C h 2^{m \alpha} \leq C h 2^{m \alpha} .
\end{aligned}
$$

Thus

$$
\left|I_{m}^{2}(h)\right| \leq \frac{C h 2^{m \alpha}}{1-e^{-2^{m+1} h}}=\frac{C 2^{m+1} h}{1-e^{-2^{m+1} h}} 2^{m(\alpha-1)} \leq C 2^{m(\alpha-1)} .
$$

Combining (6.4) and (6.5), one gets $\left|I_{m}(h)\right| \leq C 2^{m(\alpha-1)}$, which obviously implies that

$$
\lim _{n \rightarrow \infty} \sum_{m=0}^{n-1} I_{m}(h)=\sum_{m=0}^{\infty} I_{m}
$$

where

$$
I_{m} \triangleq \lim _{h \rightarrow 0} I_{m}(h)=2^{-m}\left[\sum_{k=1}^{2^{m}-1} k^{\alpha}+2^{m \alpha-1}-\int_{0}^{2^{m}} x^{\alpha} d x\right] .
$$

Note that $\sum_{m=0}^{\infty} I_{m}$ and $I$ are two numbers, so it suffices to show that

$$
\sum_{m=0}^{\infty} I_{m}<I
$$


To this end, we note that

$$
\begin{aligned}
2^{m} & I_{m}=\sum_{k=1}^{2^{m}-1} \int_{k-\frac{1}{2}}^{k+\frac{1}{2}}\left(k^{\alpha}-x^{\alpha}\right) d x+\int_{2^{m}-\frac{1}{2}}^{2^{m}}\left(2^{m \alpha}-x^{\alpha}\right) d x-\int_{0}^{\frac{1}{2}} x^{\alpha} d x \\
= & \sum_{k=1}^{2^{m}-1} \int_{0}^{\frac{1}{2}}\left[2 k^{\alpha}-(k+x)^{\alpha}-(k-x)^{\alpha}\right] d x+\int_{0}^{\frac{1}{2}}\left[2^{m \alpha}-\left(2^{m}-x\right)^{\alpha}\right] d x-\int_{0}^{\frac{1}{2}} x^{\alpha} d x \\
= & \alpha \sum_{k=1}^{2^{m}-1} \int_{0}^{\frac{1}{2}} \int_{0}^{x}\left[(k-y)^{\alpha-1}-(k+y)^{\alpha-1}\right] d y d x \\
& +\alpha \int_{0}^{\frac{1}{2}} \int_{0}^{x}\left[\left(2^{m}-y\right)^{\alpha-1}-2^{m(\alpha-1)}+2^{m(\alpha-1)}\right] d y d x-\frac{1}{(1+\alpha) 2^{1+\alpha}} \\
= & \alpha(1-\alpha) \sum_{k=1}^{2^{m}-1} \int_{0}^{\frac{1}{2}} \int_{0}^{x} \int_{-y}^{y}(k+z)^{\alpha-2} d z d y d x \\
& +\alpha(1-\alpha) \int_{0}^{\frac{1}{2}} \int_{0}^{x} \int_{0}^{y}\left(2^{m}-z\right)^{\alpha-2} d z d y d x+\frac{\alpha}{8} 2^{m(\alpha-1)}-\frac{1}{(1+\alpha) 2^{1+\alpha}} \\
\leq & \frac{\alpha(1-\alpha)}{8}\left[\sum_{k=1}^{2^{m}-1} \int_{k-\frac{1}{2}}^{k+\frac{1}{2}} z^{\alpha-2} d z+\int_{2^{m}-\frac{1}{2}}^{2^{m}}\right. \\
\leq & \frac{\alpha(1-\alpha)}{8} \int_{\frac{1}{2}}^{\infty} z^{\alpha-2} d z+\frac{\alpha}{8} 2^{m(\alpha-1)}-\frac{1}{(1+\alpha) 2^{1+\alpha}} \\
= & \frac{\alpha}{8} 2^{1-\alpha}+\frac{\alpha}{8} 2^{m(\alpha-1)}-\frac{1}{(1+\alpha) 2^{1+\alpha}} .
\end{aligned}
$$

Therefore,

$$
\begin{aligned}
& \sum_{m=0}^{\infty} I_{m} \leq \sum_{m=0}^{\infty} 2^{-m}\left[\frac{\alpha}{8} 2^{1-\alpha}+\frac{\alpha}{8} 2^{m(\alpha-1)}-\frac{1}{(1+\alpha) 2^{1+\alpha}}\right] \\
& =\frac{\alpha}{2^{1+\alpha}}+\frac{\alpha}{2\left(4-2^{\alpha}\right)}-\frac{1}{(1+\alpha) 2^{\alpha}} \\
& \leq \frac{1}{2^{1+\alpha}}+\frac{1}{4}-\frac{1}{(1+1) 2^{\alpha}}=\frac{1}{4}<\frac{1}{2} \leq \frac{1}{(1+\alpha)\left(2^{\alpha}-1\right)}=I .
\end{aligned}
$$

That proves (6.6), whence (5.8), for $\alpha<1$.

Proof of Lemma 4.1. Recall (4.5). Let, by abusing the notation $r$,

$$
x \triangleq \frac{h^{2}}{\sigma^{2}+b h}, \quad y \triangleq \frac{b h}{\sigma^{2}+b h}, \quad r(x, y) \triangleq x+\sqrt{x^{2}+2 x+y^{2}} .
$$

Then $y \leq 1$,

$$
\begin{aligned}
\frac{\sqrt{\sigma^{2}+b h}}{\sqrt{\sigma^{2}+b h}+b}\left[\frac{\sigma_{h}}{h}-\frac{\sigma}{h}\right] & =\frac{\sqrt{x}}{\sqrt{x}+y}\left[\frac{\sqrt{2}}{\sqrt{\log \frac{1+r(x, y)}{1-y} \log \frac{1+r(x, y)}{1+y}}}-\sqrt{\frac{1-y}{x}}\right] \\
& \triangleq g_{1}(x, y),
\end{aligned}
$$


and

$$
\frac{b_{h}}{h}-\frac{b}{h}=\frac{\log \frac{1+y}{1-y}}{\log \frac{1+r(x, y)}{1-y} \log \frac{1+r(x, y)}{1+y}}-\frac{y}{x} \triangleq g_{2}(x, y) .
$$

We want to show that $g_{1}$ and $g_{2}$ are bounded. Obviously both functions are continuous in $(0, \infty) \times(0,1)$. So it suffices to estimate them on the boundaries. First, it is easy to check that

$$
\left\{\begin{array}{l}
g_{1}(\infty, y)=g_{2}(\infty, y)=0, \quad \forall y \in(0,1), \\
g_{1}(x, 0)=\frac{\sqrt{2}}{\log \left(1+x+\sqrt{x^{2}+2 x}\right)}-\frac{1}{\sqrt{x}}, \quad g_{2}(x, 0)=0, \quad \forall x \in(0, \infty), \\
g_{1}(x, 1)=0, \quad g_{2}(x, 1)=\frac{1}{\log (1+x)}-\frac{1}{x}, \quad \forall x \in(0, \infty), \\
\quad \lim _{x \rightarrow \infty, y \rightarrow 0} g_{1}(x, y)=\lim _{x \rightarrow \infty, y \rightarrow 0} g_{2}(x, y)=0, \\
\lim _{x \rightarrow \infty, y \rightarrow 1} g_{1}(x, y)=\lim _{x \rightarrow \infty, y \rightarrow 1} g_{2}(x, y)=0 .
\end{array}\right.
$$

Next, for fixed $y \in(0,1]$ and small $x>0$, we have

$$
r(x, y)=y+\frac{1+y}{y} x-\frac{1-y^{2}}{2 y^{3}} x^{2}+o\left(x^{2}\right),
$$

where $o\left(x^{2}\right)$ denotes variables with higher order than $x^{2}$ as $x \rightarrow 0$. Then

$$
\log \frac{1+r(x, y)}{1+y}=\log \left(1+\frac{r-y}{1+y}\right)=\frac{x}{y}-\frac{x^{2}}{2 y^{3}}+o\left(x^{2}\right) .
$$

Therefore, for $\forall y \in(0,1)$, we have

$$
\left\{\begin{array}{l}
g_{1}(0, y)=\lim _{x \rightarrow 0} \frac{\sqrt{2 x}}{y \sqrt{\left[\log \frac{1+y}{1-y}+o(1)\right]\left[\frac{x}{y}+o(x)\right]}}-\frac{\sqrt{1-y}}{y}=\frac{\sqrt{2}}{\sqrt{y \log \frac{1+y}{1-y}}}-\frac{\sqrt{1-y}}{y} \\
g_{2}(0, y)=\lim _{x \rightarrow 0}\left[\frac{\log \frac{1+y}{1-y}}{\left[\log \frac{1+y}{1-y}+\frac{x}{y}+o(x)\right]\left[\frac{x}{y}-\frac{x^{2}}{2 y^{3}}+o\left(x^{2}\right)\right]}-\frac{y}{x}\right]=\frac{1}{2 y}-\frac{1}{\log \frac{1+y}{1-y}}
\end{array}\right.
$$

Moreover, one can easily prove that

$$
\lim _{x \rightarrow 0, y \rightarrow 1} g_{1}(x, y)=0, \quad \lim _{x \rightarrow 0, y \rightarrow 1} g_{2}(x, y)=\frac{1}{2} .
$$

Finally, we assume $(x, y) \rightarrow(0,0)$. Let

$$
\tilde{x} \triangleq \frac{2 y}{1-y}, \quad \tilde{y} \triangleq \frac{r-y}{1+y}, \quad \tilde{z} \triangleq \tilde{x}+\tilde{y}+\tilde{x} \tilde{y} .
$$

Then $\tilde{x}, \tilde{y}, \tilde{z}>0,(\tilde{x}, \tilde{y}, \tilde{z}) \rightarrow(0,0,0)$, and

$$
x=\frac{\tilde{y} \tilde{z}}{(2+\tilde{x})(1+\tilde{y})}, \quad y=\frac{\tilde{x}}{2+\tilde{x}} .
$$


We shall use Taylor expansions to estimate $g_{1}$ and $g_{2}$. Note that

$$
\frac{1+y}{1-y}=1+\tilde{x}, \quad \frac{1+r}{1+y}=1+\tilde{y}, \quad \frac{1+r}{1-y}=1+\tilde{z} .
$$

One can calculate straightforwardly

$$
\begin{aligned}
& g_{1}(x, y)=\frac{1}{\sqrt{x}+y}\left[\frac{\sqrt{2 x}}{\sqrt{\log \frac{1+r}{1-y} \log \frac{1+r}{1+y}}}-\sqrt{1-y}\right] \\
& =\frac{1}{\sqrt{x}+y}\left[\frac{\sqrt{2 \tilde{y} \tilde{z}}}{\sqrt{(2+\tilde{x})(1+\tilde{y}) \log (1+\tilde{z}) \log (1+\tilde{y})}}-\frac{\sqrt{2}}{\sqrt{2+\tilde{x}}}\right] \\
& =\frac{\sqrt{2}}{\sqrt{2+\tilde{x}}} \frac{1}{\sqrt{x}+y}\left[\frac{\sqrt{\tilde{y} \tilde{z}}}{\sqrt{(1+\tilde{y}) \log (1+\tilde{z}) \log (1+\tilde{y})}}-1\right] \\
& =\frac{1+o(1)}{\sqrt{x}+y}\left[\frac{\tilde{y} \tilde{z}}{(1+\tilde{y}) \log (1+\tilde{z}) \log (1+\tilde{y})}-1\right] \\
& \div\left[\frac{\sqrt{\tilde{y} \tilde{z}}}{\sqrt{(1+\tilde{y}) \log (1+\tilde{z}) \log (1+\tilde{y})}}+1\right] \\
& =\frac{1+o(1)}{2(\sqrt{x}+y)} \frac{\tilde{y} \tilde{z}-(1+\tilde{y}) \log (1+\tilde{z}) \log (1+\tilde{y})}{(1+\tilde{y}) \log (1+\tilde{z}) \log (1+\tilde{y})} \\
& =\frac{1+o(1)}{2(\sqrt{x}+y)} \frac{\tilde{y} \tilde{z}-(1+\tilde{y}) \tilde{z}\left[1-\frac{1}{2} \tilde{z}+o(\tilde{z})\right] \tilde{y}\left[1-\frac{1}{2} \tilde{y}+o(\tilde{y})\right]}{\tilde{y} \tilde{z}[1+o(1)]} \\
& =\frac{1+o(1)}{2} \frac{\frac{1}{2}(\tilde{z}-\tilde{y})+o(\tilde{y}+\tilde{z})}{\sqrt{\frac{\tilde{y} \tilde{z}}{2}}+\frac{\tilde{x}}{2}}=\frac{1+o(1)}{2} \frac{\tilde{x}+o(\tilde{x}+\tilde{y})}{\sqrt{2 \tilde{y} \tilde{z}+\tilde{x}}} .
\end{aligned}
$$

Since $\frac{\tilde{x}+\tilde{y}}{\sqrt{2 \tilde{y} z}+\tilde{x}} \leq 1$, we get, for $x, y$ small enough,

$$
\left|g_{1}(x, y)\right| \leq \frac{1+o(1)}{2} \leq 1 .
$$

Similarly, we have

$$
\begin{aligned}
& g_{2}(x, y)=\frac{\log (1+\tilde{x})}{\log (1+\tilde{z}) \log (1+\tilde{y})}-\frac{\tilde{x}(1+\tilde{y})}{\tilde{y} \tilde{z}} \\
& =\frac{\tilde{y} \tilde{z} \log (1+\tilde{x})-\tilde{x}(1+\tilde{y}) \log (1+\tilde{y})[\log (1+\tilde{x})+\log (1+\tilde{y})]}{\tilde{y} \tilde{z} \log (1+\tilde{z}) \log (1+\tilde{y})} \\
& =\frac{[\tilde{y} \tilde{z}-\tilde{x}(1+\tilde{y}) \log (1+\tilde{y})] \log (1+\tilde{x})-\tilde{x}(1+\tilde{y}) \log ^{2}(1+\tilde{y})}{\tilde{y} \tilde{z} \log (1+\tilde{z}) \log (1+\tilde{y})} \\
& =\frac{\left[\tilde{y} \tilde{z}-\tilde{x} \tilde{y}\left(1+\frac{1}{2} \tilde{y}+o(\tilde{y})\right)\right] \tilde{x}\left(1-\frac{1}{2} \tilde{x}+o(\tilde{x})\right)-\tilde{x}(1+\tilde{y}) \tilde{y}^{2}(1-\tilde{y}+o(\tilde{y}))}{\tilde{y}^{2} \tilde{z}^{2}(1+o(1))} \\
& =\frac{\tilde{x} \tilde{y}^{2}(1+o(\tilde{x}))-\tilde{x} \tilde{y}^{2}(1+o(\tilde{y}))}{\tilde{y}^{2} \tilde{z}^{2}(1+o(1))}=\frac{\tilde{x}[o(\tilde{x})+o(\tilde{y})]}{\tilde{z}^{2}(1+o(1))}=o(1) .
\end{aligned}
$$

By (6.7) -6.11), we prove that $g_{1}$ and $g_{2}$ are bounded. That completes the proof. 


\section{ACKNOWLEDGMENTS}

The author would like to thank N. Krylov and N. Jain for very helpful discussions.

\section{REFERENCES}

[1] Barles, G. and Jakobsen, E. (2002), On the convergence rate of approximation schemes for Hamilton-Jacobi-Bellman equations, M2AN Math. Model. Numer. Anal., 36, 33-54. MR1916291 (2003h:65142)

[2] Barles, G. and Jakobsen, E. (2005), Error bounds for monotone approximation schemes for Hamilton-Jacobi-Bellman equations, SIAM J. Numer. Anal., 43, 540-558. MR 2177879

[3] Barles, G. and Souganidis, P. (1991), Convergence of approximation schemes for fully nonlinear second order equations, Asymp. Anal., 4, 271-283. MR.1115933 (92d:35137)

[4] Bonnans, J. and Zidani, H. (2003), Consistency of generalized finite difference schemes for the stochastic HJB equation, SIAM J. Numer. Anal., 41, 1008-1021 (electronic). MR2005192 (2004i:49061)

[5] Brezzi, F., Hughes, T., Marini, L., Russo, A., and Suli, E. (1999), A priori error analysis of residual-free bubbles for advection-diffusion problems, SIAM J. Numer. Anal., 36, 1933-1948 (electronic). MR 1712145 (2000i:65171)

[6] Brezzi, F., Hauke, G., Marini, L., and Sangalli, G. (2003), Link-cutting bubbles for the stabilization of convection-diffusion-reaction problems, Dedicated to Jim Douglas, Jr. on the occasion of his 75th birthday. Math. Models Methods Appl. Sci., 13, 445-461. MR:1977635 (2004f:65163)

[7] Brezzi, F., Marini, D., and Suli, E. (2000), Residual-free bubbles for advection-diffusion problems: the general error analysis, Numer. Math., 85, 31-47. MR1751366 (2001e:65166)

[8] Brooks, A. and Hughes, T. (1982), Streamline upwind/Petrov-Galerkin formulations for convection dominated flows with particular emphasis on the incompressible Navier-Stokes equations, Comput. Methods Appl. Mech. Engrg., 32, 199-259. MR0679322 (83k:76005)

[9] Burger, R. and Karlsen, K. (2001), On some upwind difference schemes for the phenomenological sedimentation-consolidation model, J. Engrg. Math., 41, 145-166. MR.1866604 (2002h:76090)

[10] Camilli, F. and Falcone, M. (1995), An approximation scheme for the optimal control of diffusion processes, RAIRO Modél. Math. Anal. Numér., 29, 97-122. MR.1326802 (96a:49033)

[11] Dong, H. and Krylov, N. (2005), On the rate of convergence of finite-difference approximations for degenerate linear parabolic equations with $C^{1}$ and $C^{2}$ coefficients, Electron. J. Differential Equations, No. 102. MR2162263

[12] Evje, S. and Karlsen, K. (2000), Monotone difference approximations of BV solutions to degenerate convection-diffusion equations, SIAM J. Numer. Anal., 37, 1838-1860 (electronic). MR.1766850 (2001g:65110)

[13] Jakobsen, E. (2003), On the rate of convergence of approximation schemes for Bellman equations associated with optimal stopping time problems, Math. Models Methods Appl. Sci. (M3AS), 13, 613-644. MR.1978929 (2004g:49054)

[14] Karatzas, I. and Shreve, S.E. (1987), Brownian Motion and Stochastic Calculus, Springer. MR0917065 (89c:60096)

[15] Karlsen, K., Risebro, N., and Towers, J. (2002), Upwind difference approximations for degenerate parabolic convection-diffusion equations with a discontinuous coefficient, IMA J. Numer. Anal., 22, 623-664. MR:1937244(2003i:65071)

[16] Krylov, N. (1998), On the rate of convergence of finite-difference approximations for Bellman's equations, St. Petersburg Math. J., 9, 639-650. MR1466804 (98h:49033)

[17] Krylov, N. (2000), On the rate of convergence of finite-difference approximations for Bellman's equations with variable coefficients, Proba. Theory and Relat. Fields, 117, 1-16. MR1759507 (2001j:65134)

[18] Krylov, N. (2005), On the rate of convergence of finite-difference approximations for Bellman equations with Lipschitz coefficients, Appl. Math. Optim., 52, 3, 365-399. MR2174020

[19] Kushner, H. and Dupuis, P. (1992), Numerical methods for stochastic control problems in continuous time, Springer-Verlag, New York. MR.1217486 (94e:93005)

[20] Menaldi, J. (1989), Some estimates for finite difference approximations, SIAM J. Control Optim., 27, 579-607. MR0993288(90m:65137) 
[21] Revuz, D. and Yor, M. (1991), Brownian Motion and Continuous Martingales, Springer. MR.1083357 (92d:60053)

[22] Talay, D. and Tubaro, L. (1991), Expansion of the global error for numerical schemes solving stochastic differential equations, Stochastic Anal. Appl., 8, 483-509. MR1091544(92e:60124)

Department of Mathematics, University of Southern California, 3620 Vermont Ave., KAP 108, Los Angeles, California 90089

E-mail address: jianfenz@usc.edu 